Preprint JINR E2-98-56

\title{
Description of the higher massless irreducible integer spins in the BRST approach
}

\author{
A. Pashnevf \\ and M. Tsulaia门 \\ JINR-Bogoliubov Theoretical Laboratory, \\ 141980 Dubna, Moscow Region, Russia
}

\begin{abstract}
The BRST approach is applied to the description of irreducible massless higher spins representations of the Poincare group in arbitrary dimensions. The total system of constraints in such theory includes both the first and the second class constraints. The corresponding nilpotent BRST charge contains terms up to the seventh degree in ghosts.
\end{abstract}

Submitted to Modern Physics Letters A

\footnotetext{
*E-mail: pashnev@thsun1.jinr.dubna.su

${ }^{\dagger}$ E-mail: tsulaia@thsun1.jinr.dubna.su
} 


\section{Introduction}

The lagrangians, describing irreducible higher spins and guaranteeing the absence of unphysical degrees of freedom must have very special structure and admit some gauge invariance both in the massive and massless cases [1]-[10]. Along with basic fields such lagrangians in general include additional fields. Some of them are auxiliary, others can be gauged away. The role of these fields is to single out the irreducible representation of the Poincare group. The massless case is investigated more extensively and has an elegant description in terms of a single tensor field with vanishing second trace [4].

The rank - $n$ symmetrical tensor field $\Phi_{\mu_{1} \mu_{2} \cdots \mu_{n}}^{(n)}(x)$ describing the irreducible higher spin $n$ must satisfy the following system of equations

$$
\begin{aligned}
& \left(\partial_{\mu}^{2}-m_{n}\right) \Phi_{\mu_{1} \mu_{2} \cdots \mu_{n}}^{(n)}(x)=0, \\
& \partial_{\mu} \Phi_{\mu \mu_{2} \cdots \mu_{n}}^{(n)}(x)=0, \\
& \Phi_{\mu \mu \mu_{3} \cdots \mu_{n}}^{(n)}(x)=0 .
\end{aligned}
$$

They correspond to the mass shell, transversality and tracelessness conditions for the field $\Phi_{\mu_{1} \mu_{2} \cdots \mu_{n}}^{(n)}(x)$. In an auxiliary Fock space, which naturally leads to the description of higher rank symmetrical tensor fields, all these conditions appear to be some constraints. The total system of constrains, corresponding to the equations (1.1)-(1.3), contains, in general, only one first class constraint (mass shell condition) and two pairs of second class constraints. In the massless case $\left(m_{n}=0\right)$ only two constraints, corresponding to the tracelessness of the field, are of the second class.

The BRST approach to the construction of the lagrangians, from which all the equations (1.1)-(1.3) follow, is very powerful. It automatically leads to appearance of all auxiliary fields in the lagrangian. In the massless case the BRST charge for the system of only first class constraints, corresponding to the equations (1.1) - (11.2) was constructed in [11]. The lagrangian describes the infinite tower of massless higher spin particles, infinitely degenerated on each spin level. The goal of the present paper is to include in the BRST charge the additional second class constraints, which delete the extra states and lead to the irreducibility conditions (1.3).

The methods of such construction were discussed in [12]- [14]. With the help of additional variables one can modify the second class constraints in such a way that they become commuting, i.e the first class. At the same time the number of physical degrees of freedom for both systems does not change if the number of additional variables coincides with the number of second class constraints.

On the other hand, the BRST charge for the second class constraints in some cases can be constructed using the method of dimensional reduction. In [16] the system of massive higher spins satisfying equations (1.1) - (1.2) was described in the framework of the BRST approach. The corresponding BRST charge is nilpotent and has a very special structure. In particular, the modified constraints have the algebra, which is not closed. Nevertheless, the nontrivial structure of trilinear terms 
in ghosts in the BRST charge compensates this defect and makes the BRST charge to be nilpotent.

In the second part of the paper we illustrate the method of BRST construction in the simple case of one first class (mass shell) and two second class constraints which implements tracelessness of the fields. The same procedure is applied in the third part of the paper to the total system of constraints, describing the massless irreducible representations of Poincare group in arbitrary $D$ - dimensional space time. The resulting nilpotent BRST charge includes in a very special way the terms up to the seventh degree in ghosts fields. The Fronsdal lagrangian [⿴囗十 is then obtained from the BRST quantized lagrangian after the partial gauge fixing.

\section{A Toy Model}

As it was mentioned in the Introduction, for description of all higher spins simultaneously it is convenient to introduce auxiliary Fock space generated by creation and annihilation operators $a_{\mu}^{+}, a_{\mu}$ with vector Lorentz index $\mu=0,1,2, \ldots D-1$, satisfying the following commutation relations

$$
\left[a_{\mu}, a_{\nu}^{+}\right]=-g_{\mu \nu}, g_{\mu \nu}=\operatorname{diag}(1,-1,-1, \ldots,-1) .
$$

The general state of the Fock space

$$
|\Phi\rangle=\sum \Phi_{\mu_{1} \mu_{2} \cdots \mu_{n}}^{(n)}(x) a_{\mu_{1}}^{+} a_{\mu_{2}}^{+} \cdots a_{\mu_{n}}^{+}|0\rangle
$$

depends on space-time coordinates $x_{\mu}$ and its components $\Phi_{\mu_{1} \mu_{2} \cdots \mu_{n}}^{(n)}(x)$ are tensor fields of rank $n$ in the space-time of arbitrary dimension $D$. The norm of states in this Fock space is not positively definite due to the negative sign in the commutation relation (2.1) for time components of creation and annihilation operators. It means, that physical states must satisfy some constraints to have positive norm.

To describe the irreducible massless higher spins we must take into account the following constraints [16]:

$$
\begin{array}{ll}
L_{0}=-p_{\mu}^{2}, & \\
L_{1}=p_{\mu} a_{\mu}, & L_{1}^{+}=p_{\mu} a_{\mu}^{+}, \\
L_{2}=\frac{1}{2} a_{\mu} a_{\mu}, & L_{2}^{+}=\frac{1}{2} a_{\mu}^{+} a_{\mu}^{+} .
\end{array}
$$

Indeed, quantizing the theory à la Gupta - Bleuler, we impose only part of the whole system of constraints on the physical states

$$
\left.\left.\left.L_{0} \mid \text { Phys }\right\rangle=L_{1} \mid \text { Phys }\right\rangle=L_{2} \mid \text { Phys }\right\rangle=0 .
$$

These equations lead to the correct system of equations for component fields $\Phi_{\mu_{1} \mu_{2} \cdots \mu_{n}}^{(n)}(x)$. In what follows we will show, that BRST quantization of the system with constraints (2.3)-(2.5) is equivalent to the Gupta - Bleuler quantization. 
The constraints (2.3)-(2.5) form the algebra:

$$
\begin{aligned}
& {\left[L_{1}, L_{-2}\right]=-L_{-1}, \quad\left[L_{-1}, L_{2}\right]=L_{1}} \\
& {\left[L_{1}, L_{-1}\right]=L_{0}, \quad\left[L_{2} \cdot L_{-2}\right]=-a_{\mu}^{+} a_{\mu}+\frac{D}{2} \equiv G_{0} .}
\end{aligned}
$$

It means that the second class constraints $L_{ \pm 2}$ must be included in the BRST charge. Following the line of [12] - [14] one might try to transform these constraints into the commuting ones by introducing additional degrees of freedom. This procedure is rather simple for the classical case when the Poisson brackets are used instead of commutators. It leads to the finite system of differential equations which can be solved without troubles. In the quantum case the corresponding system of equations is infinite due to accounting of repeated commutators.

In this section we describe the modification of the procedure of [12] - 14] in the simplified case of one first class constraint $L_{0}$ (2.3) and two second class constraints $L_{2}, L_{-2}$ (2.5). It gives the structure of the BRST charge analogous to one derived in [16 by dimensional reduction. To illustrate the BRST - approach to this simple system, we introduce the set of anticommuting variables $\eta_{0}, \eta_{2}, \eta_{2}^{+}$, having ghost number one and corresponding momenta $\mathcal{P}_{0}, \mathcal{P}_{2}^{+}, \mathcal{P}_{2}$, with commutation relations:

$$
\left\{\eta_{0}, \mathcal{P}_{0}\right\}=\left\{\eta_{2}, \mathcal{P}_{2}^{+}\right\}=\left\{\eta_{2}^{+}, \mathcal{P}_{2}\right\}=1
$$

We modify our system of constraints by introduction of additional operator $b$ together with its conjugate $b^{+}:\left[b, b^{+}\right]=-1$. By the analogy with [16], we consider two modified constraints $\tilde{L}_{2}$ and $\tilde{L}_{-2}$ :

$$
\begin{aligned}
\tilde{L}_{2} & =L_{2}+X_{1} b, \\
\tilde{L}_{-2} & =L_{-2}+b^{+} X_{1}, \\
X_{1} & =\sqrt{G_{0}-b^{+} b} .
\end{aligned}
$$

In spite of the fact, that these operators do not have a closed algebra,

$$
\begin{aligned}
{\left[\tilde{L}_{2}, \tilde{L}_{-2}\right] } & =-b^{+} X_{2} \tilde{L}_{2}-\tilde{L}_{-2} X_{2} b+b^{+} X_{2}^{2} b \\
X_{2} & =\sqrt{G_{0}-b^{+} b}-\sqrt{G_{0}-b^{+} b+2}
\end{aligned}
$$

the special structure of the last two terms in the BRST charge

$$
Q=\eta_{2}^{+} \tilde{L}_{2}+\eta_{2} \tilde{L}_{2}^{+}+\eta_{0} L_{0}+\eta_{2}^{+} \eta_{2} \mathcal{P}_{2} b^{+} X_{2}-\eta_{2}^{+} \mathcal{P}_{2}^{+} \eta_{2} X_{2} b
$$

leads to its nilpotency.

Consider the total Fock space generated by creation operators $a_{\mu}^{+}, b^{+}, \eta_{0}, \eta_{2}^{+}, \mathcal{P}_{2}^{+}$. The BRST - invariant lagrangian in such Fock space can be written as:

$$
L=-\int d \eta_{0}\langle\chi|Q| \chi\rangle
$$




$$
|\chi\rangle=\left|S_{1}\right\rangle+\eta_{2}^{+} \mathcal{P}_{2}^{+}\left|S_{2}\right\rangle+\eta_{0} \mathcal{P}_{2}^{+}\left|S_{3}\right\rangle,
$$

with vectors $\left|S_{i}\right\rangle$ having ghost number zero and depending only on bosonic creation operators $a_{\mu}^{+}, b^{+}$

$$
\left|S_{i}\right\rangle=\sum \phi_{i ; \mu_{1}, \mu_{2}, \ldots \mu_{n}}^{k}(x) a_{\mu_{1}}^{+} a_{\mu_{2}}^{+} \ldots a_{\mu_{n}}^{+}\left(b^{+}\right)^{k}|0\rangle .
$$

After the integration over the $\eta_{0}$ we get the following lagrangian in terms of $\left|S_{i}\right\rangle$

$$
\begin{aligned}
L= & -\left\langle S_{1}\left|L_{0}\right| S_{1}\right\rangle+\left\langle S_{2}\left|L_{0}\right| S_{2}\right\rangle+\left\langle S_{1}\left|L_{2}^{+}+b^{+} X_{1}\right| S_{3}\right\rangle+ \\
& \left\langle S_{3}\left|L_{2}+X_{1} b\right| S_{1}\right\rangle-\left\langle S_{2}\left|L_{2}+X_{1,2} b\right| S_{3}\right\rangle-\left\langle S_{3}\left|L_{2}^{+}+b^{+} X_{1,2}\right| S_{2}\right\rangle,
\end{aligned}
$$

where we have introduced the notation

$$
X_{1, n}=\sqrt{G_{0}-b^{+} b+n} .
$$

Owing to the nilpotency of the BRST - charge - $Q^{2}=0$, the lagrangian (2.16) is invariant under the transformation

$$
\delta|\chi\rangle=Q|\Lambda\rangle
$$

with $|\Lambda\rangle=\mathcal{P}_{2}^{+}|\lambda\rangle$. Now it is straightforward to write the component form of the gauge transformations

$$
\begin{aligned}
\delta\left|S_{1}\right\rangle & =\left(L_{2}^{+}+b^{+} X_{1}\right)|\lambda\rangle \\
\delta\left|S_{2}\right\rangle & =\left(L_{2}+X_{1,2} b\right)|\lambda\rangle, \\
\delta\left|S_{3}\right\rangle & =L_{0}|\lambda\rangle .
\end{aligned}
$$

and the lagrangian equations of motion as well:

$$
\begin{aligned}
L_{0}\left|S_{1}\right\rangle & =\left(L_{2}^{+}+b^{+} X_{1}\right)\left|S_{3}\right\rangle \\
L_{0}\left|S_{2}\right\rangle & =\left(L_{2}+X_{1,2} b\right)\left|S_{3}\right\rangle \\
\left(L_{2}+X_{1} b\right)\left|S_{1}\right\rangle & =\left(L_{2}^{+}+b^{+} X_{1,2}\right)\left|S_{2}\right\rangle
\end{aligned}
$$

One can prove that the gauge freedom (2.22)-(2.24) is sufficient to eliminate the fields $\left|S_{2}\right\rangle$ and $\left|S_{3}\right\rangle$ and to kill the $b^{+}$dependence in $\left|S_{1}\right\rangle$. First we eliminate the field $\left|S_{3}\right\rangle$ with the help of transformation (2.24). Then there will be residual gauge invariance with the parameter $\left|\lambda^{\prime}\right\rangle$ under the condition

$$
L_{0}\left|\lambda^{\prime}\right\rangle=0
$$

Eliminating of field $\left|S_{2}\right\rangle$ with the help of the equation $\left|S_{2}\right\rangle+\left(L_{2}+X_{1,2} b\right)\left|\lambda^{\prime}\right\rangle=0$, which is consistent with (2.28) and equations of motion, the new residual parameter $\left|\lambda^{\prime \prime}\right\rangle$ will satisfy two conditions $L_{0}\left|\lambda^{\prime \prime}\right\rangle=\left(L_{2}+X_{1,2} b\right)\left|\lambda^{\prime \prime}\right\rangle=0$. With the help of this parameter all the fields $\left|S_{1, k}\right\rangle$, having $k$-th degree of operator $b^{+}$-

$$
\left|S_{1, k}\right\rangle \equiv \phi_{1 ; \mu_{1}, \mu_{2}, \ldots \mu_{n}}^{k} a_{\mu_{1}}^{+} a_{\mu_{2}}^{+} \ldots a_{\mu_{n}}^{+}\left(b^{+}\right)^{k}|0\rangle \text {. }
$$


except $\left|S_{1,0}\right\rangle$ can be eliminated as well. One can easily see, that the lagrangian (2.19) falls into a sum of pieces, each connecting the vectors with different $n$ and $k$ numbers of operators $a_{\mu}^{+}$and $b^{+}$in $\left|S_{1}\right\rangle$. Let us denote such fields as $\Phi_{n}^{k}$. Then the following set of them are connected in the lagrangian:

$$
\Phi_{n}^{0}, \Phi_{n-2}^{1}, \ldots, \Phi_{n-2\left[\frac{n}{2}\right]}^{\left[\frac{n}{2}\right]},
$$

where $\left[\frac{n}{2}\right]$ stands for the integer part of the number $\frac{n}{2}$. Starting from the end of the chain (2.30) with the help of transformation (2.22) one can delete step by step all its components except the first one $\Phi_{n}^{0}$. Therefore the following conditions on the reduced field $\left|S_{1,0}\right\rangle$ are obtained:

$$
L_{0}\left|S_{1,0}\right\rangle=L_{2}\left|S_{1,0}\right\rangle=0
$$

The second of the conditions (2.31) means tracelessness of the wavefunctions $\phi_{1 ; \mu_{1}, \mu_{2}, \ldots \mu_{n}}^{0}$. The first one simply implies masslessness of the field. Note the lack of transversality condition and consequent presence of ghosts in the spectrum of our simple model. In order to kill them, in the next section we will include in the consideration the constraints $L_{ \pm 1}$ as well.

\section{$3 \quad$ Irreducible massless higher spins}

As it was shown in [17], all constraints in the total system (2.3)-(2.5) can be converted into the first class constraints by introduction of two additional operators $b_{1}, b_{2}$ together with their conjugates $b_{1}^{+}, b_{2}^{+},\left[b_{1}, b_{1}^{+}\right]=-1,\left[b_{2}, b_{2}^{+}\right]=1$. The modified constraints are: $\tilde{L}_{0}=L_{0}, \tilde{L}_{ \pm 1}=\tilde{L}_{ \pm 1}, \tilde{L}_{2}=L_{2}+b_{1}^{+} b_{2}, \tilde{L}_{-2}=L_{-2}+b_{2}^{+} b_{1}$ and $\tilde{G}_{0}=G_{0}+b_{2}^{+} b_{2}+b_{1}^{+} b_{1}$. Note the appearance of one additional constraint $\tilde{G}_{0}$, which manifestly depends on the dimensionality $D$ of space - time. This dependence leads to consistent description of higher irreducible spins only if $D$ is even.

Now we will construct the BRST charge, lagrangian etc. along the line of preceding Section, by introducing only one pair of additional operators $b, b^{+},\left[b, b^{+}\right]=-1$.

Now we introduce larger set of anticommuting variables $\eta_{0}, \eta_{1}, \eta_{1}^{+} \eta_{2}, \eta_{2}^{+}$having ghost number one and corresponding momenta $\mathcal{P}_{0}, \mathcal{P}_{1}^{+}, \mathcal{P}_{1}, \mathcal{P}_{2}^{+}, \mathcal{P}_{2}$ with commutation relations:

$$
\left\{\eta_{0}, \mathcal{P}_{0}\right\}=\left\{\eta_{1}, \mathcal{P}_{1}^{+}\right\}=\left\{\eta_{1}^{+}, \mathcal{P}_{1}\right\}=\left\{\eta_{2}, \mathcal{P}_{2}^{+}\right\}=\left\{\eta_{2}^{+}, \mathcal{P}_{2}\right\}=1
$$

The following nilpotent BRST charge corresponds to the total system of constraints (2.3)-(2.5)

$$
\begin{aligned}
Q= & \eta_{0} L_{0}+\eta_{1} L_{1}^{+}+\eta_{2} L_{2}^{+}+\eta_{1}^{+} L_{1}+\eta_{2}^{+} L_{2}+\eta_{2}^{+} X_{1} b+\eta_{2} b^{+} X_{1}- \\
& \eta_{1}^{+} \eta_{1} \mathcal{P}_{0}-\eta_{1}^{+} \mathcal{P}_{1}^{+} \eta_{2}+\eta_{2}^{+} \eta_{1} \mathcal{P}_{1}-\eta_{2}^{+} \mathcal{P}_{2}^{+} \eta_{2} X_{2} b+\eta_{2}^{+} \eta_{2} \mathcal{P}_{2} b^{+} X_{2}- \\
& \eta_{1}^{+} \eta_{2}^{+} \mathcal{P}_{1} X_{3} b+\mathcal{P}_{1}^{+} \eta_{1} \eta_{2} b^{+} X_{3}+\eta_{2}^{+} \mathcal{P}_{1}^{+} \eta_{1} X_{3} b-\eta_{1}^{+} \eta_{2} \mathcal{P}_{1} b^{+} X_{3}- \\
& \eta_{2}^{+} \mathcal{P}_{1}^{+} \mathcal{P}_{2}^{+} \eta_{1} \eta_{2} X_{4} b-\eta_{1}^{+} \eta_{2}^{+} \eta_{2} \mathcal{P}_{1} \mathcal{P}_{2} b^{+} X_{4}-\eta_{1}^{+} \eta_{2}^{+} \mathcal{P}_{2}^{+} \eta_{2} \mathcal{P}_{1} X_{4} b- \\
& \eta_{2}^{+} \mathcal{P}_{1}^{+} \eta_{1} \eta_{2} \mathcal{P}_{2} b^{+} X_{4}+\eta_{1}^{+} \eta_{2}^{+} \mathcal{P}_{1}^{+} \eta_{1} \mathcal{P}_{1} X_{5} b+\eta_{1}^{+} \mathcal{P}_{1}^{+} \eta_{1} \eta_{2} \mathcal{P}_{1} b^{+} X_{5}- \\
& \eta_{1}^{+} \eta_{2}^{+} \mathcal{P}_{1}^{+} \mathcal{P}_{2}^{+} \eta_{1} \eta_{2} \mathcal{P}_{1} X_{6} b+\eta_{1}^{+} \eta_{2}^{+} \mathcal{P}_{1}^{+} \eta_{1} \eta_{2} \mathcal{P}_{1} \mathcal{P}_{2} b^{+} X_{6}
\end{aligned}
$$


where

$$
\begin{aligned}
& X_{1}=\sqrt{-1+G_{0}-b^{+} b} \\
& X_{1, n} \equiv \sqrt{-1+G_{0}-b^{+} b+n} \\
& X_{2}=X_{1}-X_{1,2} \\
& X_{3}=-X_{1}+X_{1,1}, \\
& X_{4}=+X_{1}-X_{1,1}-X_{1,2}+X_{1,3}, \\
& X_{5}=-X_{1}+2 X_{1,1}-X_{1,2}, \\
& X_{6}=+X_{1}-2 X_{1,1}+2 X_{1,3}-X_{1,4} .
\end{aligned}
$$

Note the appearance of additional -1 in the definition of $X_{1}$ as a result of inclusion of constraints $L_{ \pm 1}$ into BRST charge.

As in the toy model, the modified constraints $\tilde{L}_{2}=L_{2}+X_{1} b, \tilde{L}_{-2}=L_{-2}+b^{+} X_{1}$ have nonclosed algebra. The consequence of this fact is the appearance in the BRST charge of the special terms up to the seventh degree in the ghosts.

Again, the BRST - invariant lagrangian can be written as in the case of the toy model

$$
-L=\int d \eta_{0}\langle\chi|Q| \chi\rangle
$$

where $|\chi\rangle$ has now more complicated form:

$$
\begin{aligned}
|\chi\rangle= & \left|S_{1}\right\rangle+\eta_{1}^{+} \mathcal{P}_{1}^{+}\left|S_{2}\right\rangle+\eta_{2}^{+} \mathcal{P}_{2}^{+}\left|S_{3}\right\rangle+\eta_{1}^{+} \mathcal{P}_{2}^{+}\left|S_{4}\right\rangle+ \\
& \eta_{2}^{+} \mathcal{P}_{1}^{+}\left|S_{5}\right\rangle+\eta_{1}^{+} \eta_{2}^{+} \mathcal{P}_{1}^{+} \mathcal{P}_{2}^{+}\left|S_{6}\right\rangle+\eta_{0} \mathcal{P}_{1}^{+}\left|A_{1}\right\rangle+ \\
& \eta_{0} \mathcal{P}_{2}^{+}\left|A_{2}\right\rangle+\eta_{0} \eta_{1}^{+} \mathcal{P}_{1}^{+} \mathcal{P}_{2}^{+}\left|A_{3}\right\rangle+\eta_{0} \eta_{2}^{+} \mathcal{P}_{1}^{+} \mathcal{P}_{2}^{+}\left|A_{4}\right\rangle
\end{aligned}
$$

with vectors $\left|S_{i}\right\rangle$ and $\left|A_{i}\right\rangle$ having ghost number zero and depending only on bosonic creation operators $a_{\mu}^{+}, b^{+}$.

Integration over the $\eta_{0}$ leads to the component form of (3.4):

$$
\begin{aligned}
-L= & \left\langle A_{1}|| A_{1}\right\rangle-\left\langle A_{2}|| S_{2}\right\rangle+\left\langle A_{3}|| S_{3}\right\rangle-\left\langle A_{4}|| A_{4}\right\rangle-\left\langle S_{2}|| A_{2}\right\rangle+\left\langle S_{3}|| A_{3}\right\rangle+ \\
& \left\langle A_{1}\left|L_{2}^{+}\right| S_{4}\right\rangle+\left\langle A_{1}\left|L_{1}^{+}\right| S_{2}\right\rangle-\left\langle A_{1}\left|L_{1}\right| S_{1}\right\rangle+\left\langle A_{2}\left|L_{2}^{+}\right| S_{3}\right\rangle+\left\langle A_{2}\left|L_{1}^{+}\right| S_{5}\right\rangle- \\
& \left\langle A_{2}\left|L_{2}\right| S_{1}\right\rangle+\left\langle A_{3}\left|L_{2}^{+}\right| S_{6}\right\rangle-\left\langle A_{3}\left|L_{1}\right| S_{5}\right\rangle+\left\langle A_{3}\left|L_{2}\right| S_{2}\right\rangle-\left\langle A_{4}\left|L_{1}^{+}\right| S_{6}\right\rangle- \\
& \left\langle A_{4}\left|L_{1}\right| S_{3}\right\rangle+\left\langle A_{4}\left|L_{2}\right| S_{4}\right\rangle-\left\langle S_{1}\left|L_{2}^{+}\right| A_{2}\right\rangle-\left\langle S_{1}\left|L_{1}^{+}\right| A_{1}\right\rangle+\left\langle S_{1}\left|L_{0}\right| S_{1}\right\rangle+ \\
& \left\langle S_{2}\left|L_{2}^{+}\right| A_{3}\right\rangle-\left\langle S_{2}\left|L_{0}\right| S_{2}\right\rangle+\left\langle S_{2}\left|L_{1}\right| A_{1}\right\rangle-\left\langle S_{3}\left|L_{1}^{+}\right| A_{4}\right\rangle-\left\langle S_{3}\left|L_{0}\right| S_{3}\right\rangle+ \\
& \left\langle S_{3}\left|L_{2}\right| A_{2}\right\rangle+\left\langle S_{4}\left|L_{2}^{+}\right| A_{4}\right\rangle-\left\langle S_{4}\left|L_{0}\right| S_{5}\right\rangle+\left\langle S_{4}\left|L_{2}\right| A_{1}\right\rangle-\left\langle S_{5}\left|L_{1}^{+}\right| A_{3}\right\rangle- \\
& \left\langle S_{5}\left|L_{0}\right| S_{4}\right\rangle+\left\langle S_{5}\left|L_{1}\right| A_{2}\right\rangle+\left\langle S_{6}\left|L_{0}\right| S_{6}\right\rangle-\left\langle S_{6}\left|L_{1}\right| A_{4}\right\rangle+\left\langle S_{6}\left|L_{2}\right| A_{3}\right\rangle+ \\
& \left\langle A_{1}\left|b^{+} X_{1,1}\right| S_{4}\right\rangle+\left\langle A_{2}\left|b^{+} X_{1,2}\right| S_{3}\right\rangle-\left\langle A_{2}\left|X_{1} b\right| S_{1}\right\rangle+\left\langle A_{3}\left|b^{+} X_{1,4}\right| S_{6}\right\rangle+ \\
& \left\langle A_{3}\left|X_{1,2} b\right| S_{2}\right\rangle+\left\langle A_{4}\left|X_{1,3} b\right| S_{4}\right\rangle-\left\langle S_{1}\left|b^{+} X_{1}\right| A_{2}\right\rangle+\left\langle S_{2}\left|b^{+} X_{1,2}\right| A_{3}\right\rangle+ \\
& \left\langle S_{3}\left|X_{1,2} b\right| A_{2}\right\rangle+\left\langle S_{4}\left|b^{+} X_{1,3}\right| A_{4}\right\rangle+\left\langle S_{4}\left|X_{1,1} b\right| A_{1}\right\rangle+\left\langle S_{6}\left|X_{1,4} b\right| A_{3}\right\rangle
\end{aligned}
$$

The BRST gauge invariance

$$
\delta|\chi\rangle=Q|\lambda\rangle
$$


with the most general parameter $|\lambda\rangle$, having ghost number -1

$$
|\lambda\rangle=\mathcal{P}_{1}^{+}\left|\lambda_{1}\right\rangle+\mathcal{P}_{2}^{+}\left|\lambda_{2}\right\rangle+\eta_{1}^{+} \mathcal{P}_{1}^{+} \mathcal{P}_{2}^{+}\left|\lambda_{3}\right\rangle+\eta_{2}^{+} \mathcal{P}_{1}^{+} \mathcal{P}_{2}^{+}\left|\lambda_{4}\right\rangle+\eta_{0} \mathcal{P}_{1}^{+} \mathcal{P}_{2}^{+}\left|\lambda_{5}\right\rangle
$$

means, in turn, the invariance of the lagrangian (3.6) under the following transformations:

$$
\begin{aligned}
\delta\left|S_{1}\right\rangle & =L_{1}^{+}\left|\lambda_{1}\right\rangle+L_{2}^{+}\left|\lambda_{2}\right\rangle+b^{+} X_{1}\left|\lambda_{2}\right\rangle \\
\delta\left|S_{2}\right\rangle & =-\left|\lambda_{2}\right\rangle+L_{1}\left|\lambda_{1}\right\rangle+L_{2}^{+}\left|\lambda_{3}\right\rangle+b^{+} X_{1,2}\left|\lambda_{3}\right\rangle \\
\delta\left|S_{3}\right\rangle & =\left|\lambda_{3}\right\rangle+L_{2}\left|\lambda_{2}\right\rangle-L_{1}^{+}\left|\lambda_{4}\right\rangle+X_{1,2} b\left|\lambda_{2}\right\rangle \\
\delta\left|S_{4}\right\rangle & =-\left|\lambda_{5}\right\rangle+L_{1}\left|\lambda_{2}\right\rangle-L_{1}^{+}\left|\lambda_{3}\right\rangle \\
\delta\left|S_{5}\right\rangle & =+L_{2}^{+}\left|\lambda_{4}\right\rangle+L_{2}\left|\lambda_{1}\right\rangle+b^{+} X_{1,3}\left|\lambda_{4}\right\rangle+X_{1,1} b\left|\lambda_{1}\right\rangle \\
\delta\left|S_{6}\right\rangle & =-L_{2}\left|\lambda_{3}\right\rangle-X_{1,4} b\left|\lambda_{3}\right\rangle+L_{1}\left|\lambda_{4}\right\rangle \\
\delta\left|A_{1}\right\rangle & =+L_{2}^{+}\left|\lambda_{5}\right\rangle+L_{0}\left|\lambda_{1}\right\rangle+b^{+} X_{1,1}\left|\lambda_{5}\right\rangle \\
\delta\left|A_{2}\right\rangle & =-L_{1}^{+}\left|\lambda_{5}\right\rangle+L_{0}\left|\lambda_{2}\right\rangle \\
\delta\left|A_{3}\right\rangle & =+L_{0}\left|\lambda_{3}\right\rangle-L_{1}\left|\lambda_{5}\right\rangle \\
\delta\left|A_{4}\right\rangle & =+L_{0}\left|\lambda_{4}\right\rangle-L_{2}\left|\lambda_{5}\right\rangle-X_{1,3} b\left|\lambda_{5}\right\rangle
\end{aligned}
$$

Since (3.7) is unaffected by the following change of the gauge parameter $\delta|\lambda\rangle=Q|\omega\rangle$, with $|\omega\rangle=\mathcal{P}_{1}^{+} \mathcal{P}_{2}^{+}\left|\omega_{1}\right\rangle$, one of the parameters $\left|\lambda_{i}\right\rangle$ in (3.8) is inessential. In what follows we choose the gauge, where $\left|\lambda_{5}\right\rangle=0$.

Combining the equations of motion

$$
\begin{array}{ll}
S 1: & -L_{2}^{+}\left|A_{2}\right\rangle-L_{1}^{+}\left|A_{1}\right\rangle+L_{0}\left|S_{1}\right\rangle-b^{+} X_{1,0}\left|A_{2}\right\rangle=0, \\
S 2: & -\left|A_{2}\right\rangle+L_{2}^{+}\left|A_{3}\right\rangle-L_{0}\left|S_{2}\right\rangle+L_{1}\left|A_{1}\right\rangle+b^{+} X_{1,2}\left|A_{3}\right\rangle=0, \\
S 3: & \left|A_{3}\right\rangle-L_{1}^{+}\left|A_{4}\right\rangle-L_{0}\left|S_{3}\right\rangle+L_{2}\left|A_{2}\right\rangle+X_{1,2} b\left|A_{2}\right\rangle=0, \\
S 4: & L_{2}^{+}\left|A_{4}\right\rangle-L_{0}\left|S_{5}\right\rangle+L_{2}\left|A_{1}\right\rangle+b^{+} X_{1,3}\left|A_{4}\right\rangle+X_{1,1} b\left|A_{1}\right\rangle=0 \\
S 5: & -L_{1}^{+}\left|A_{3}\right\rangle-L_{0}\left|S_{4}\right\rangle+L_{1}\left|A_{2}\right\rangle=0, \\
S 6: & L_{0}\left|S_{6}\right\rangle-L_{1}\left|A_{4}\right\rangle+L_{2}\left|A_{3}\right\rangle+X_{1,4} b\left|A_{3}\right\rangle=0, \\
A 1: & \left|A_{1}\right\rangle+L_{2}^{+}\left|S_{4}\right\rangle+L_{1}^{+}\left|S_{2}\right\rangle-L_{1}\left|S_{1}\right\rangle+b^{+} X_{1,1}\left|S_{4}\right\rangle=0, \\
A 2: & L_{2}^{+}\left|S_{3}\right\rangle+L_{1}^{+}\left|S_{5}\right\rangle-L_{2}\left|S_{1}\right\rangle+b^{+} X_{1,2}\left|S_{3}\right\rangle-X_{1,0} b\left|S_{1}\right\rangle-\left|S_{2}\right\rangle=0, \\
A 3: & L_{2}^{+}\left|S_{6}\right\rangle-L_{1}\left|S_{5}\right\rangle+L_{2}\left|S_{2}\right\rangle+b^{+} X_{1,4}\left|S_{6}\right\rangle+X_{1,2} b\left|S_{2}\right\rangle+\left|S_{3}\right\rangle=0, \\
A 4: & -\left|A_{4}\right\rangle-L_{1}^{+}\left|S_{6}\right\rangle-L_{1}\left|S_{3}\right\rangle+L_{2}\left|S_{4}\right\rangle+X_{1,3} b\left|S_{4}\right\rangle=0,
\end{array}
$$

and the gauge transformations (3.9) one can prove, that only essential field is the $b^{+}$- independent part of $\left|S_{1}\right\rangle$, which satisfies all needed equations and describes at each level $n$ exactly one massless representation of the $D$ - dimensional Poincare group with spin $n$. Instead of straightforward choice of gauge, leading to these results, we will show, how to connect our approach to already known elegant description of massless higher spins in terms of one symmetrical tensor field with vanishing second trace 四]. 
Using the gauge freedom (3.9) with parameters $\left|\lambda_{i}\right\rangle,(i=1,2,3,4)$, one can eliminate all fields except $\left|S_{1}\right\rangle,\left|S_{2}\right\rangle$ and $\left|A_{1}\right\rangle$. Moreover, as in the case of toy model, one can kill the $b^{+}$- dependence of these fields. We denote the remaining fields as $\left|S_{1,0}\right\rangle,\left|S_{2,0}\right\rangle$ and $\left|A_{1,0}\right\rangle$. The system of equations of motion (3.10), except the ones for the fields $\left|S_{1,0}\right\rangle$, and $\left|S_{2,0}\right\rangle$ which lead to the dynamical equations, now reads

$$
\begin{gathered}
L_{2}\left|A_{1,0}\right\rangle=0, \\
\left|A_{1,0}\right\rangle+L_{1}^{+}\left|S_{2,0}\right\rangle-L_{1}\left|S_{1,0}\right\rangle=0, \\
L_{2}\left|S_{1,0}\right\rangle+\left|S_{2,0}\right\rangle=0, \\
L_{2}\left|S_{2,0}\right\rangle=0,
\end{gathered}
$$

with residual invariance

$$
\delta\left|S_{1,0}\right\rangle=L_{1}^{+}\left|\lambda_{1}\right\rangle, \delta\left|S_{2,0}\right\rangle=L_{1}\left|\lambda_{1}\right\rangle, \delta\left|A_{1,0}\right\rangle=L_{0}\left|\lambda_{1}\right\rangle,
$$

where parameter $\left|\lambda_{1}\right\rangle$ is restricted by the condition

$$
L_{2}\left|\lambda_{1}\right\rangle=0 .
$$

Using the equations (3.12) and (3.13) one can express $\left|S_{2,0}\right\rangle$ and $\left|A_{1,0}\right\rangle$ through $\left|S_{1,0}\right\rangle$ and insert them into (3.6). The lagrangian now depends only on the field $\left|S_{1,0}\right\rangle$ and takes the following form 15]

$$
\begin{aligned}
-L= & \left\langle S_{1,0}\right| L_{0}-L_{1}^{+} L_{1}-L_{1}^{+} L_{1}^{+} L_{2}-L_{2}^{+} L_{1} L_{1} \\
& -2 L_{0} L_{2}^{+} L_{2}-L_{2}^{+} L_{1}^{+} L_{1} L_{2}\left|S_{1,0}\right\rangle
\end{aligned}
$$

The field $\left|S_{1,0}\right\rangle$, as a consequence of the equations (3.13) and (3.14), is restricted by the condition

$$
L_{2} L_{2}\left|S_{1,0}\right\rangle=0,
$$

making (3.11) to be identity. After taking the expansion

$$
\left|S_{1,0}\right\rangle=\phi_{\mu_{1}, \mu_{2}, \ldots \mu_{n}} a_{\mu_{1}}^{+} a_{\mu_{2}}^{+} \ldots a_{\mu_{n}}^{+}|0\rangle
$$

we find that the lagrangian (3.17) in terms of the fields $\phi_{\mu_{1}, \mu_{2}, \ldots \mu_{n}}$ coincides with the one given by Fronsdal [4]. As the consequence of the condition (3.18) the field $\phi_{\mu_{1}, \mu_{2}, \ldots \mu_{n}}$ has vanishing second trace $\phi_{\nu \nu \rho \rho \mu_{5}, \mu_{6}, \ldots \mu_{n}}$ and the lagrangian is invariant under the transformation

$$
\delta \phi_{\mu_{1}, \mu_{2}, \ldots \mu_{n}}=\partial_{\left\{\mu_{1}\right.} \lambda_{\left.\mu_{2} \mu_{3} \ldots \mu_{n}\right\}}
$$

with constrained parameter $\lambda_{\nu \nu \mu_{3} \ldots \mu_{n}}=0$. 


\section{Conclusions}

In this paper we have applied the BRST approach to the description of irreducible massless higher spins. The nilpotent BRST charge was constructed and corresponding lagrangian, containing along with basic field some auxiliary fields was derived. When the gauge in the model is partially fixed, the resulting lagrangian coincides with the lagrangian of [4]. It would be interesting to generalize the procedure to the case of halfinteger spins [5]. It seems to be possible, since the approaches to the description of integer and halfinteger spins are similar to each other. The main difference will be the presence of odd constraints, leading to the Dirac equation, and, corresponding,appearance of the bosonic ghosts in the BRST charge.

Acknowledgments. This investigation has been supported in part by the Russian Foundation of Fundamental Research, grants 96-02-17634 and 96-02-18126, joint grant RFFR-DFG 96-02-00180G, and INTAS, grants 93-127-ext, 96-0308, 96-0538, 94-2317 and grant of the Dutch NWO organization.

\section{References}

[1] E.S.Fradkin. JETP, 20 (1950) 27

[2] L.P.H.Singh, C.R.Hagen. Phys.Rev., D9 (1974) 898; Ibid D9 (1974) 910

[3] S.J.Chang. Phys.Rev., 161 (1967) 1308

[4] C.Fronsdal. Phys.Rev., D18 (1978) 3624

[5] J.Fang, C.Fronsdal. Phys.Rev., D18 (1978) 3630

[6] B.de Wit, D.Z. Freedman. Phys.Rev., D21 (1980) 358

[7] T. Curtright. Phys.Lett., B85 (1979) 219

[8] M.A. Vasiliev. Sov.J.Nucl.Phys., 32 (1980) 855 (439 in english translation)

[9] C. Aragone, S. Deser. Nucl.Phys., B170 (1980) 329

[10] I. Tyutin, M. Vasiliev., Theor.Math.Phys., 113 (1997) 45, hep-th/9704132

[11] S.Ouvry, J.Stern., Phys.Lett., B177 (1986) 335

[12] L.D. Faddeev, S.L. Shatashvili. Phys.Lett., B167 (1986) 225

[13] I.A. Batalin, E.S. Fradkin. Nucl.Phys., B279 (1987) 514

[14] E.T. Egoryan, R.P. Manvelyan. Theor. Math.Phys., 94 (1993) 241 
[15] A.I.Pashnev. Theor.Math.Phys., 78 (1989) 424

[16] A. Pashnev, M. Tsulaia., Mod.Phys.Lett., A12 (1997) 861

[17] A. Pashnev, M. Tsulaia., Talk at the D.V. Volkov memorial conference, Kharkov, 1997 